\title{
Criminologie
}

\section{De la sécurité privée à l'armée de destruction massive. La " bande armée " et la criminalité de masse}

\section{Samuel Tanner}

Volume 45, numéro 1, printemps 2012

Violence politique et conflits armés

URI : https://id.erudit.org/iderudit/1008375ar

DOI : https://doi.org/10.7202/1008375ar

Aller au sommaire du numéro

\section{Éditeur(s)}

Les Presses de l’Université de Montréal

ISSN

0316-0041 (imprimé)

1492-1367 (numérique)

Découvrir la revue

Citer cet article

Tanner, S. (2012). De la sécurité privée à l'armée de destruction massive. La « bande armée » et la criminalité de masse. Criminologie, 45(1), 29-49.

https://doi.org/10.7202/1008375ar
Résumé de l'article

Dans cet article, nous nous intéressons à la mobilisation et la participation d'une bande armée - les Scorpions - dans les événements qui se sont produits en ex-Yougoslavie, durant les années 1990. Si l'étude de cette figure centrale des crimes de masse est nécessaire à la compréhension de ce phénomène et, ultimement, à sa prévention, elle se justifie aussi d'un point de vue épistémologique : plutôt que d'ancrer une réflexion sur l'individu et ses motivations, démarche courante pour une criminologie dominante centrée sur les préoccupations sécuritaires des sociétés occidentales, nous postulons que c'est à partir du groupe et de la nature éminemment collective et politique de cette forme de criminalité extrême que la réflexion sur les dynamiques d'élimination de populations entières doit s'amorcer. Cette démarche permet la genèse de connaissances plus spécifiques et un regard nouveau sur la criminalité de masse.
Ce document est protégé par la loi sur le droit d'auteur. L'utilisation des services d’Érudit (y compris la reproduction) est assujettie à sa politique d'utilisation que vous pouvez consulter en ligne.

https://apropos.erudit.org/fr/usagers/politique-dutilisation/ 


\title{
De la sécurité privée à l'armée de destruction massive. La « bande armée » et la criminalité de masse
}

\author{
Samuel Tanner \\ Professeur adjoint \\ École de criminologie, Université de Montréal \\ samuel.tanner@umontreal.ca
}

RÉSUMÉ - Dans cet article, nous nous intéressons à la mobilisation et la participation d'une bande armée - les Scorpions - dans les événements qui se sont produits en ex-Yougoslavie, durant les années 1990. Si l'étude de cette figure centrale des crimes de masse est nécessaire à la compréhension de ce phénomène et, ultimement, à sa prévention, elle se justifie aussi d'un point de vue épistémologique: plutôt que d'ancrer une réflexion sur l'individu et ses motivations, démarche courante pour une criminologie dominante centrée sur les préoccupations sécuritaires des sociétés occidentales, nous postulons que c'est à partir du groupe et de la nature éminemment collective et politique de cette forme de criminalité extrême que la réflexion sur les dynamiques d'élimination de populations entières doit s'amorcer. Cette démarche permet la genèse de connaissances plus spécifiques et un regard nouveau sur la criminalité de masse.

MOTS-CLÉs - Violence de masse, ex-Yougoslavie, Scorpions, bandes armées, radicalisation, sécurité privée.

\section{La «bande armée » comme objet d'une criminologie nouvelle?}

La criminalité de guerre est restée un objet marginal de préoccupation des criminologues. Pourtant, si l'on songe en termes de sécurité des populations et de pertes humaines, elle représente le phénomène qui a probablement engendré le plus de victimes au cours du dernier siècle. C'est sur les auteurs de la violence de guerre que notre intérêt porte dans le cadre de cette contribution, et plus spécifiquement sur les 
«bandes armées». Ce terme désigne des petits groupes de gens en armes qui apparaissent dans le contexte d'un conflit armé et qui agissent à l'intersection d'une criminalité civile, ou de droit commun (vols, trafics, extorsion), et d'une criminalité politique, ou étatique, dont le crime de guerre, le crime contre l'humanité, ou encore le génocide constituent les formes les plus extrêmes. Dans les événements qui ont marqué les Balkans, au cours des années 1990, et qui serviront de trame à notre propos, les Tigres de feu Željko Ražnjatovic, alias Arkan ${ }^{1}$, ou encore les Scorpions, en représentent des exemples types.

Ces formations se composent d'acteurs d'une violence de proximité, ou de face à face. Ils sont directement confrontés - tant physiquement que temporellement - aux conséquences de leurs actes. Aussi, et pour les distinguer des «criminels de bureau», notion empruntée à Hannah Arendt, et généralement qualifiés d'exécutants, nous aurons affaire ici à des exécuteurs ${ }^{2}$. Puisqu'en vertu de cette définition tout auteur d'une violence de proximité peut être désigné comme exécuteur, il est important d'opérer une seconde distinction entre exécuteurs réguliers versus irréguliers, ceux-ci composant essentiellement les bandes armées. Est considéré irrégulier tout individu participant activement à une violence de guerre, sans pour autant qu'il soit membre permanent d'une bureaucratie d'État ou d'une force armée légale: police, paramilitaires, ou armée régulière ${ }^{3}$. L'irrégulier est un acteur non étatique, ou privé, et participe à la guerre sur un mode d'appoint ${ }^{4}$.

1. Déjà reconnu comme une grande figure du banditisme serbe et international avant les guerres des années 1990, Arkan a eu un rôle crucial dans les conflits armés des années 1990, notamment en mettant sur pied les Tigres, essentiellement mobilisés à partir des supporters de son club de football, l'Étoile rouge de Belgrade, disciplinés et entraînés aux techniques de la guerre (Stewart, 2007). Arkan a été assassiné le 15 janvier 2000 à Belgrade.

2. L'exécutant renvoie à une catégorie épistémique spécifique d'acteurs de la violence, à savoir le fonctionnaire d'une machine administrative vouée à l'élimination d'une population. La bureaucratie tend à diffuser la responsabilité de ses «serviteurs», et ainsi étouffer leur sens moral, provoquant un état d'indifférenciation à l'égard des victimes (Bauman, 1989). Hannah Arendt parle dans ce cas d'individus qui ne pensent plus, ou sont incapables de juger par eux-mêmes la situation dans laquelle ils évoluent. Adolf Eichmann est probablement le cas paradigmatique de l'exécutant, tel qu'analysé par la philosophe (Arendt, 1966). Par opposition, l'exécuteur a, quant à lui, les deux pieds dans la violence et il agit directement sur le terrain. Ses armes ne sont pas tant le stylo et le formulaire administratif que le couteau ou la mitraillette. D'un point de vue épistémologique, l'exécuteur renvoie à un objet différent de l'exécutant.

3. Plus spécifiquement, et au moment où il commet l'acte de violence, l'acteur n'est pas employé sur une base permanente au sein d'une force armée régulière qui l'habilite légalement à faire usage de la force légitime.

4. Sur la question des acteurs non étatiques de la violence, on renvoie le lecteur aux travaux de Fowler (2005), Weinstein (2007), ou encore Schulz et Dew (2006). 
Dans une réflexion sur la criminalité extrême, Stéphane LemanLanglois s'interroge: "Peut-on prétendre comprendre le crime sans le mégacrime?» (Leman-Langlois, 2006: 34). La réponse est non, mais encore faut-il se donner les moyens et les outils conceptuels de saisir ce phénomène complexe. C'est dans ce questionnement que s'inscrit cette contribution, qui traite de la participation de bandes armées dans les événements qui se sont produits en ex-Yougoslavie durant les années 1990. Plus particulièrement: quelles sont les logiques de mobilisation et de participation des bandes armées à la violence de masse, telle qu'elle s'est produite sur ce territoire? Si l'étude de cette figure centrale d'une forme de destruction de l'Homme des plus radicales est nécessaire à sa compréhension et, ultimement, à sa prévention, elle se justifie aussi d'un point de vue épistémologique: plutôt que de cibler l'individu et ses motivations, démarche courante pour une criminologie dominante centrée sur les préoccupations sécuritaires des sociétés occidentales (Brodeur, 2007), nous postulons que c'est à partir du groupe et de la nature éminemment collective et politique de cette forme de criminalité extrême que la réflexion sur les dynamiques d'élimination de populations entières doit s'amorcer. Cette démarche permettra la genèse de nouvelles connaissances et un regard nouveau sur la criminalité de masse.

\section{La criminalité de masse comme produit d'une radicalisation}

Davantage qu'un «big bang» intentionnel (Browning, 2007) dont la genèse reposerait exclusivement sur les épaules d'une élite politique et militaire qui «par le haut» ordonnerait, par l'entremise d'une chaîne de commandement, l'élimination d'une population, certains ont montré qu'il faut considérer la criminalité de masse comme un processus cumulatif de radicalisation, sous-tendu par un dispositif complexe d'extermination (Mommsen, 1989; Shaw, 2007). Ian Kershaw (1998) affirme que les pratiques de destruction sous-tendant l'Holocauste n'auraient pas tant été le produit ultime de bureaucrates et officiers nazis obéissant strictement à l'autorité, mais plutôt le résultat d'un processus de radicalisation. En particulier, affirme-t-il, elles auraient résulté de l'exploitation d'un cadre formulé par Hitler en des termes généraux et laissant un espace suffisamment grand à l'interprétation par les différents échelons hiérarchiques du dispositif nazi pour la mise en œuvre de ses 
volontés. Partant du principe que seule l'action efficace - et s'inscrivant en direction des volontés du Führer - était récompensée d'une promotion au sein du pouvoir et de la bureaucratie nazis, c'est l'initiative des subordonnés du dispositif, en compétition les uns les autres, et poursuivant une série de décisions à la pièce, qui aurait engendré une logique de sélection du pire en matière de pratiques et de stratégies d'élimination des populations, et ce, jusqu'à leur extermination physique (Kershaw, 1998). La naissance des chambres à gaz en aurait constitué l'expression extrême. Indépendamment d'une idéologie raciste, ou antisémite, c'est le souci de mettre en œuvre les volontés d'Hitler, ayant force de loi et apparaissant comme autant d'impératifs d'actions, qui expliquerait le zèle et le systématisme avec lesquels ces populations ont été exterminées (Arendt, 2003). Ce paradigme suggère que la destruction d'une population résulterait d'un «jeu» de miroirs et de profit mutuel entre acteurs des pouvoirs central et périphérique. D'une part, les objectifs et volontés des acteurs centraux sont formulés de manière suffisamment floue, ce qui leur permet de nier toute participation de l'État à des actions criminelles et d'afficher un déni plausible de toute implication dans le crime. Cependant, et d'autre part, l'esprit de ces objectifs et volontés est assez clair pour assurer la garantie à ceux qui les mettent à exécution qu'ils obtiendront des avantages considérables, qu'ils soient matériels, symboliques ou politiques. Le principe du jeu de miroirs constitue un cadre pertinent pour interpréter une grande proportion des logiques qui ont sous-tendu l'élimination des populations en ex-Yougoslavie, durant les années 1990. Il permet notamment de saisir la participation de bandes armées à ces événements et c'est précisément l'une d'elles, les Scorpions, qui va mobiliser notre intérêt.

\section{Une étude de cas: les Scorpions et la violence de masse en Bosnie-Herzégovine}

La bande armée des Scorpions a été portée à l'attention du public par la diffusion d'une bande vidéo, tournée par l'un de ses membres, documentant l'exécution de six civils musulmans, à Trnovo, près de Sarajevo, les 16 et 17 juillet 1995. Ces images, qui ont provoqué une onde de choc et d'indignation, ont permis l'arrestation quasi immédiate de leurs protagonistes et la conduite de leur procès, en octobre 2005, à la Chambre spéciale chargée des crimes de guerre au sein du tribunal de district de Belgrade (War Crimes Prosecutor's Office, 2005). Cinq 
d'entre eux ont été jugés: Slobodan Medic, alias Boco (condamné à vingt ans de prison); son frère, Aleksandar Medic (condamné à cinq ans de prison); Pera Petrasevic (condamné à treize ans de prison); Branisalv Medic (condamné à vingt ans de prison); et enfin Aleksandar Vukov (acquitté pour manque de preuves). C'est basé sur cette affaire, et notamment sur un document audiovisuel décrivant les Scorpions (Majdak, 2007), ainsi que sur des retranscriptions d'audiences du procès (War Crimes Chamber, 20065), que l'analyse qui suit repose. Le document audiovisuel est d'une valeur toute particulière, puisqu'il y est question du témoignage de deux anciens membres repentis des Scorpions, Goran Stoparic et Dusko Kosanovic, qui possédaient une connaissance intime de la bande. C'est particulièrement le cas de Kosanovic qui était responsable de filmer et documenter tous les faits et gestes de ses collègues. Il s'agit d'un corpus inédit ${ }^{6}$.

Au-delà de son usage juridique, ce matériel représente une grande valeur pour le chercheur en sciences sociales. Il documente, de l'intérieur, les faits et gestes de cette unité et ouvre une fenêtre originale sur les dynamiques sociales qui la caractérisent ${ }^{7}$. Il permet de saisir les perceptions et représentations des exécuteurs, certes, dans le cadre de leur propre défense, et offre autant d'informations pertinentes pour dégager une compréhension de la participation de ces acteurs à la violence de masse. Rappelons que, selon le concept d'action socialement orientée, élaboré par Max Weber (1921), toute action est liée à une signification. Les acteurs agissent en vertu d'un cadre référentiel, ou normatif, qui informe leurs préférences, leurs représentations et, ultimement, guide leurs actions. À son tour, ce cadre s'élabore et prend forme au gré des relations de groupe et des interactions en leur sein. En effet, note Weber, il est nécessaire de distinguer deux types de rationalité. On relève d'une part une rationalité qui s'oriente en fonction des individus qui entourent immédiatement l'acteur (Wertrationalität) et

5. Retranscriptions d'audiences couvrant la période du 20 décembre 2005 au 5 juillet 2006.

6. L'ensemble des citations tirées de ce documentaire a été retranscrit sous forme de verbatim à partir des sous-titrages de la bande.

7. Selon Howard S. Becker, «La difficulté la plus récurrente que rencontre l'étude scientifique de la déviance est le manque de données solides, l'insuffisance des faits et des informations sur lesquels nous pourrions fonder nos théories. [...] Il n'y a pas assez d'études qui fournissent des données sur le mode d'existence des déviants, selon le point de vue de ceux-ci [...]» (Becker, 1985: 189-190). En cela, ce matériel tend à répondre aux exigences du sociologue. 
qui se démarque d'une rationalité guidée par une valeur absolue (religion, nationalisme, éthique, etc.) (Zweckrationalität). Dans les études qui traitent du comportement génocidaire, cette seconde rationalité a largement orienté l'analyse: les tueurs agiraient alors en vertu d'un absolu (qu'il s'agisse par exemple de la pureté raciale ou d'un idéal nationaliste). Or, les acteurs se conduisent aussi en relation à la contingence immédiate dans laquelle ils évoluent, et des relations qu'ils entretiennent avec leur environnement social, politique et économique.

Au regard d'une appréhension chronologique de la participation des Scorpions dans la violence de masse, on observe deux moments distincts. Un premier s'étend de sa création, en 1992, en tant que compagnie de sécurité privée chargée de la protection d'une société d'exploitation de pétrole, en Croatie, jusqu'en 1994, date à laquelle, amorçant la seconde phase, elle se métamorphose en armée de destruction massive et participe activement aux tueries de civils. Ce n'est que dans cette seconde phase que l'unité a été nommée les Scorpions à proprement parler. Procédons à une analyse détaillée de chacun de ces moments.

«Sécurité privée » et exploitation d'une opportunité économique: 1992-1994

La genèse de cette bande armée, initialement compagnie de sécurité privée, tient d'un contexte d'opportunité politique, économique et social particulier dont il convient de rappeler les événements déterminants.

En février 1992, à la suite d'un conflit armé en Croatie, entre Croates et Serbes, qui aura duré environ une année, un accord de paix est signé entre les deux clans, mettant un terme aux combats. Or, les tensions demeurent et, malgré le déploiement de la Force de protection des Nations Unies $^{8}$ visant à maintenir la paix dans la région, chacun tente malgré tout d'assurer la sécurité de ses populations et ressources par ses propres moyens. C'est dans ce contexte qu'un service de sécurité privée est mis en place, qui est chargé de la protection d'une société d'exploitation de champs de pétrole, à Deletovci, petite ville située sur un territoire saisi par les forces serbes durant la guerre après une campagne féroce d'élimination des populations croates locales. L'acte d'accusation de l'affaire Slobodan Medic et al. indique que c'est un certain Milan 
«Mrgud» Milanovic, chef de la branche de la Sécurité d'État serbe à Novi Sad, qui aurait proposé à son ami directeur de la compagnie d'exploitation, la Naftna Industrija Republike Srpske Krajina, de mettre sur pied un service de sécurité privée, pour autant que la compagnie fournisse les ressources matérielles et financières adéquates à l'unité9. Pour ce faire, il prend contact avec un certain Slobodan Medic, alias Boco, à qui il délègue la mise sur pied d'une équipe privée de gardiens de sécurité. Ce choix s'explique par la réputation dont Boco jouissait dans la région, essentiellement comme bienfaiteur local et entrepreneur prospère, bénéficiant d'un capital important. Il avait également été repéré par les services de sécurité lors de sa participation au conflit armé entre Serbes et Croates. C'est ce qu'indique en partie le témoignage de Mrgud au procès de Slobodan Milosević :

J'ai rencontré ce jeune homme [Slobodan Medic] pour la première fois. Plusieurs personnes m'avaient parlé de lui et j'ai même demandé à Bazda [chef de la police de Serbie] s'il avait quoi que ce soit contre lui. Il m'a répondu que ce n'était pas le cas. Ce jeune homme avait à l'époque 22, 23 ans et avait montré beaucoup de compétence dans l'organisation de son travail, qu'il a poursuivi d'ailleurs jusqu'à la fin de 1996. (Le Procureur du Tribunal contre Slobodan Milosević, 2004: 27492)

C'est accompagné d'une quinzaine d'hommes que Slobodan Medic prend en charge l'organisation et la mise sur pied de la sécurité des champs de pétrole. Tous deviennent salariés à temps plein de la compagnie. Considérant l'époque, leur paie était élevée puisqu'ils touchaient deux cent cinquante deutschemarks par mois ${ }^{10}$. Dans son témoignage, Goran Stoparic précise que, contrairement à la plupart des chefs de milice d'alors, Boco n'était ni un officier entrâné ni un ancien membre de la Légion étrangère (Majdak, 2007). Il bénéficiait pourtant d'une réputation d'homme intelligent et influent dans sa communauté. Bien que simple berger, Boco était un maillon important d'un vaste réseau de contrebande de moutons et de bœufs, qui enrichissait un grand nombre d'individus, y compris des officiers locaux. Ces éléments

9. L'acte d'accusation de l'affaire Slobodan Medic et al. mentionne que:

Puisque la République de Krajina serbe [région serbe de Croatie] était engagée dans un conflit armé avec la République de Croatie, et que les champs se situaient près de la frontière le long de la rivière Bosut, le besoin est apparu de fournir du personnel afin de garder ces champs et les installations [pétrolières] situées sur ces derniers (The Prosecutor of the Tribunal vs Slobodan Medic, op. cit., notre traduction).

10. Soit environ l'équivalent d'un peu plus de 200 \$ CAN actuels. 
expliquent en grande partie le capital dont il bénéficiait. Lui et son frère, Aleksandar Medic, ainsi que certains de leurs amis proches ayant joint le service de sécurité, avaient aussi été présents sur le champ des violences à Vukovar, à la fin de l'année 1991 (Anastasijevic, 2005) et leur expérience a constitué un atout majeur dans leur nouvelle assignation. Ainsi donc, c'est un faisceau d'éléments qui distinguent Slobodan Medic du quidam et le rapprochent davantage d'autres figures de la guerre impliquées dans des activités criminelles de type «crime organisé» avant même le conflit, dont notamment Arkan, bien qu'il n'ait jamais égalé la célébrité de ce dernier.

En période de sanction économique et d'embargo, décrétés par la communauté internationale, la possession et l'exploitation des champs de pétrole ont constitué une possibilité d'enrichissement inimaginable et inégalable par les canaux réguliers d'une économie serbe à genoux. Il s'agissait d'une opportunité sans prix pour ces trois principales sphères de protagonistes, à savoir la direction de la compagnie, les autorités serbes - de Serbie comme de Croatie - et la compagnie de sécurité dirigée par Slobodan Medic. En conséquence, l'intérêt de maintenir cette entreprise et préserver cette collaboration, ou collusion, était élevé. Goran Stoparic révèle que:

Plus personne ne vivait là-bas, seulement les Scorpions. Ils étaient déployés là-bas à cause des gains importants liés aux champs de pétrole, service de la part de la compagnie pétrolière de la Krajina qui, en retour, les soutenait avec de l'argent et tout ce dont ils avaient besoin, des salaires, et je ne sais quoi d'autre. C'était ça l'accord. (Majdak, 2007, notre traduction)

Les bénéfices engendrés par cette entreprise commune étaient d'autant plus colossaux que le prix de l'essence avait atteint des sommets et que cette exploitation pétrolière assurait vingt pour cent des besoins de la Serbie. Le commerce et la contrebande du pétrole, ainsi que du bois de chêne - autre richesse de la région -, de cigarettes et de voitures volées, se faisaient sous la protection de l'unité de Slobodan Medic ainsi que sous le contrôle d'officiels haut placés au pouvoir central serbe. On identifie notamment Jovica Stanisic et Frenki Simatovic, respectivement numéro un et numéro deux du Service de sécurité d'État de la République de Serbie (Anastasijevic, 2005). Tout contributeur avait droit à sa part, et une fois les acteurs centraux «rétribués» - État serbe et hommes d'affaires locaux -, le profit demeurait suffisamment élevé pour permettre à Medic et ses hommes de s'enrichir. Il était donc dans l'intérêt commun 
de maintenir le bon fonctionnement de cette entreprise et d'assurer des dividendes maximas à chacun. Enfin, Milan Milanovic, devenu entretemps ministre adjoint de la Défense de la République serbe de Croatie garantissait la «légalité» de ces opérations, ce qui facilitait le transit des marchandises entre les frontières bosniaque et croate.

Ainsi, on observe une instrumentalisation mutuelle entre les différents acteurs qui participaient à l'entreprise de profit qui, progressivement, a prospéré et s'est cristallisée ${ }^{11}$, où les gains ont été observés tant sur le plan économique qu'en vertu d'un double jeu politique. D'une part, les autorités serbes pouvaient nier publiquement toute implication dans des activités criminelles en cours, qu'elles présentaient comme le fait de maraudeurs incontrôlables, affichant alors un engagement de surface aux accords de paix entre la Serbie et la Croatie. D'autre part, cette complicité avec le régime permettait à cette unité, ou compagnie de sécurité privée, d'accumuler du capital politique, du fait de sa proximité avec les hauts dirigeants, et gagner en influence aux yeux de la communauté locale. Parallèlement, les Scorpions ont su tirer profit des ressources et de l'instrument considérable qu'était l'État - notamment dans la liberté quasi absolue qu'il conférait à cette bande - pour mener à bien ses activités criminelles. Une logique similaire de potentialisation des dividendes entre acteurs périphériques et centraux s'observe dans le cas des Tigres, d'Arkan, et de la relation d'instrumentalisation mutuelle qu'ils entretenaient avec la clique de Slobodan Milosević (Judah, 1997; Stewart, 2007). Dans le cas des Scorpions, l'entreprise criminelle a duré jusqu'en 1995, date de l'Opération éclair, décidée par le Conseil de sécurité des Nations Unies, et la récupération par l'armée croate de la région, ainsi que la signature des Accords d'Erdut.

Lopportunité et l'exploitation d'un cadre économique et politique nécessitent également d'être appréhendées du point de vue des individus et membres de l'unité des Scorpions. À quelques exceptions près, une grande majorité d'entre eux n'avait qu'une brève expérience dans les forces serbes de Croatie (armée comme police). En effet, à part un ou

11. La cristallisation est à la sphère locale et communautaire - c'est-à-dire infra-étatique - ce que l'institutionnalisation est à la sphère étatique ou sociétale: la mise en place d'un ordre stable et permanent. Si l'institutionnalisation est corrélative à l'élaboration de règles de droit externes aux groupes qui composent la société - où tout groupe doit respecter un code criminel -, la cristallisation repose quant à elle sur des normes négociées et tacites qui résultent des interactions entre membres du groupe. Elles sont réactualisables et réactualisées en permanence par le groupe ou la communauté. 
deux individus, ex-employés d'un service municipal de police en Serbie, la grande majorité n'était pas des membres réguliers de l'armée serbe de Croatie - mise sur pied en 1991. La plupart d'entre eux, essentiellement des volontaires, ne disposaient donc que d'une expérience limitée du combat. Seuls deux témoins ont affirmé avoir appartenu à l'unité d'Arkan, les Tigres. Le reste des membres se composait de fermiers, chauffeurs, et d'un traiteur. En conséquence, dans un contexte économique difficile et à l'aube d'une guerre imminente - en Bosnie-Herzégovine -, nombreux ont été ceux qui ont préféré s'engager dans l'entreprise de sécurité privée, espérant éviter de servir dans l'armée régulière et d'être envoyés au front ${ }^{12}$. Beaucoup ont adhéré à cette compagnie de sécurité privée après y avoir été convaincus par un ami, ou un proche, déjà membre. Nous reviendrons sur ce point plus systématiquement par la suite. Relevons pour terminer que la paie, sans surprise, a constitué un atout majeur pour un grand nombre d'entre eux, les incitant à s'enrôler dans le service de sécurité de la Naftna Industrija Republike Srpske Krajina, en cette période de difficulté économique.

De 1992 à 1994, ce jeu de profit mutuel, résultant d'une opportunité économique, politique et sociale, s'est articulé autour d'une activité criminelle de faible à moyenne portée, c'est-à-dire de pratiques criminelles limitées aux trafic, extorsion et corruption. Pourtant, et en dépit de la signature des accords de paix, ces activités se sont non seulement poursuivies, mais ont également évolué vers une forme de criminalité extrême, ou à longue portée, dont les conséquences, cette fois, ont mené à des tueries de civils.

De la sécurité privée à l'armée de destruction massive: 1994-1995

Parallèlement à la gestion de la sécurité des champs de pétrole, Slobodan Medic a été désigné pour former et entraîner un groupe d'hommes au combat, au sein même de la compagnie de sécurité. C'est ce groupe qui deviendra les Scorpions, réelle armée de destruction

12. C'est une dynamique similaire à celle observée par Christopher R. Browning (1992) dans les logiques d'enrôlement des individus du $101^{\mathrm{e}}$ bataillon de la police de réserve allemande, essentiellement formée d'individus qui, initialement, souhaitaient s'enrôler dans la police, et censée demeurer sur les lignes arrières de la guerre, plutôt que dans l'armée, afin d'éviter le contact direct avec la ligne de front et la violence. 
massive. Cette formation a rapidement été rattachée au Corps de Vukovar de l'Armée de la République serbe de Krajina [il s'agit de l'armée serbe de Croatie], sous le commandement d'un général nommé Dusan Loncar. Il s'agissait d'un groupe qui complétait les rangs de l'armée en vue d'anticiper le réarmement croate. Selon les retranscriptions des audiences de l'affaire Slobodan Medic et al, le groupe aurait reçu l'ordre, à deux reprises, d'assister les unités militaires qui combattaient contre l'armée de Bosnie-Herzégovine. La première fois, en novembre 1994, à Petrovo Selo, petite bourgade croate proche de la frontière nord-est, entre la Croatie et la Bosnie, et la deuxième fois, à Velika Kladusa, dans la même région. Également, les Scorpions étaient désignés par les autorités serbes pour venir en aide à l'armée serbe de Bosnie (VRS), dans le village de Trnovo, proche de Sarajevo, en juillet 1995 (War Crimes Chamber, 2006). C'est dans cette région que l'exécution de six civils musulmans qui apparaît sur la bande vidéo citée plus haut a eu lieu.

Le lien entre les Scorpions et le gouvernement central ne fait pas de doute et il est indiscutable qu'une collusion entre les deux parties explique leur présence dans les violences de masse. Slobodan Medic affirme :

Vous savez, les gens disent toutes sortes de choses, que nous étions affiliés [...] au service de sécurité d'État serbe, au Corps de Vukovar, je n'en sais vraiment rien. Je suis profondément blessé quand je lis que c'était une unité paramilitaire. Essayez de juger par vous-mêmes si quelqu'un avait pu armer 550 hommes de sa poche, si je peux le dire ainsi, payer leur salaire et le reste. (War Crimes Chamber, 2006, audience du 21 décembre 2005, notre traduction)

Goran Stoparic prétend que l'ensemble du matériel et de l'armement était également fourni par la compagnie d'exploitation de pétrole. Il affirme :

Je connaissais les hommes, je savais qu'ils étaient tous bien équipés, je savais qu'ils avaient des contacts avec la Serbie. Sinon, une unité de l'armée de la République serbe de Krajina n'aurait pas pu avoir des jeeps modernes, de l'équipement de l'OTAN, être mieux équipée que les corps de police militaire, ça aurait été inconcevable. (Majdak, 2007, notre traduction)

Les emblèmes qui figuraient sur les tenues des membres de l'unité et qui affichaient le drapeau tricolore tendent également à confirmer qu'ils agissaient sous les ordres, du moins le consentement, des autorités de 
la République de Serbie ${ }^{13}$. Cela dit, du point de vue des acteurs de terrain, il est intéressant de relever comment ce rapport aux autorités officielles se déployait. Le témoignage de Goran Stoparic fournit des indices à ce sujet:

C'est ici que j'ai vu le général Mladic pour la première fois. Il a donné un discours. Il a dit qu'il allait avancer [environ] cinq cents mètres par heure et qu'il se foutait même des animaux domestiques jusqu'à Livno [Bosnie orientale], mais que nous devions faire attention aux civils à Livno même. J'ai réalisé qu'il n'était pas du tout intéressé à faire des prisonniers, ni même aux civils, puisqu'il avait dit qu'il se foutait des animaux domestiques. Nous étions probablement libres de tuer tout le monde. (Majdak, 2007, notre traduction)

Ce passage documente un aspect déterminant du fonctionnement de l'articulation entre pouvoir central et exécuteurs de terrain. Il s'interprète parfaitement dans le cadre théorique de radicalisation émergente, $\mathrm{du}$ jeu de pouvoir entre acteurs centraux et périphériques, dont les bandes armées, et du principe d'anticipation de la volonté du chef, présenté ci-dessus. Indiquons cependant que Mladic bénéficiait d'un charisme important aux yeux des hommes de terrain «combattant pour la nation serbe ${ }^{14} »$. Aussi, et tout comme dans le cas d'Hitler, toutes proportions gardées, les objectifs du général de l'armée serbe avaient valeur de direction et de cadre à mettre en application. Ici, l'objectif du chef est clair, du moins dans l'interprétation qu'en fait Stoparic, mais les moyens d'y parvenir demeurent indéfinis, ou énoncés en termes généraux. Ce phénomène évoque les propos d'Arendt (2003), explicités ci-dessus, relatifs à l'interprétation et la séquence d'événements qu'elle

13. Le lien qui unit les Scorpions avec les autorités serbes est précisé lors du témoignage d'un certain Tomislav Kovac, qui, jusqu'en 1995, était ministre adjoint des Affaires intérieures de la République serbe de Bosnie, et commandant de la police serbe de Bosnie. Celui-ci confirme l'envoi par l'armée serbe de Croatie d'une force armée composée de trois unités. Il s'agissait d'une compagnie de police d'Erdut (Croatie); une compagnie des Tigres d'Arkan, affiliée à l'armée serbe de Bosnie (VRS) et enfin, les Scorpions, de Deletovci, incorporés à l'armée serbe de Croatie. Kovac précise aussi que l'ensemble de ces unités agissait sous un commandement unique, et plus spécifiquement sous les ordres d'un certain Vasilje Milovic. Il ajoute qu'à ce moment précis (1994-1995), aucune unité paramilitaire, ni milice, n'était légalement permise sur le territoire, ce qui revient à conclure que les Scorpions, tout comme les Tigres, appartenaient au ministère de la Défense de Serbie, ou une quelconque institution de forces armées serbe officielle de Croatie, Serbie, ou Bosnie (War Crimes Chamber, 2006, audience du 2 décembre 2005).

14. Sur cette question, le lecteur pourra consulter Drakulic (2004), plus précisément le chapitre 11, «Punished by the Gods» (154-175). 
implique : autonomie et compétition entre acteurs qui, in fine, mènent à la radicalisation des pratiques et la destruction des populations civiles. C'est dans la relation entre les hommes de terrain et le charisme de Mladic qu'un rapport de force de type «inciter, susciter, combiner [...]» (Deleuze, 1986: 36) se déploie. On observe, de la part des subordonnés, un mouvement d'anticipation de la volonté du chef militaire, ce qui provoque une radicalisation et une violence qui, progressivement, s'émancipent ou s'autonomisent d'un cadre, ou chaîne de commandement légal. Cette articulation révèle une nouvelle forme de profit mutuel, puisque dans ce cas, et sans que les ordres ne soient directement énoncés, les acteurs périphériques, y compris les exécuteurs des bandes armées, mettent en application les volontés du pouvoir central qui, en retour, métamorphose ces individus en héros patriotiques, ou "guerriers célestes», selon la tradition nationaliste serbe (Drakulic, 2004 ; Judah, 1997). Formulés en ces termes, il ne s'agit plus tant de tueurs et de criminels, mais bien, à travers l'adoption de rôles paradigmatiques ${ }^{15}$, de protecteurs de la nation serbe. Cet exemple met en exergue une nouvelle morphologie de la cristallisation des relations entre acteurs périphériques et pouvoir central. Elle ne s'articule non plus tant exclusivement autour d'un jeu de profit mutuel économique et matériel - se confinant à une criminalité de faible et moyenne portée -, mais aussi, et désormais, autour de bénéfices mutuels politiques qui font appel à une criminalité de longue portée (élimination d'une population), transformant par la même occasion cette compagnie de sécurité privée en armée de destruction massive.

Il est également crucial de tenir compte des logiques internes aux Scorpions puisque cela permet de jeter une lumière nouvelle tant sur les modes de mobilisation de ce type d'acteurs que leur régulation interne et leur précipitation dans l'action radicale. Goran Stoparic relève que la mobilisation de la majorité des membres, ainsi que leur participation à la violence, ont résulté d'un mécanisme de contagion. Beaucoup ont adhéré à l'unité par cooptation, et parce qu'un ami, proche ou membre de la famille s'étant joint au groupe auparavant les a convaincus. Goran Stoparic affirme:

15. L'adoption de rôles paradigmatiques renvoie au mécanisme par lequel, au sein d'une population, des individus adoptent, à la suite d'un événement particulier, un rôle socialement significatif pour exprimer une attitude ou une croyance profondément ancrée (Petersen, 2001). 
Plus tard [la participation dans la violence] est devenue comme une sorte de drogue, et un homme ne pouvait s'en défaire par lui-même. Même si tu décides de ne pas aller dans une zone de combat, un ami t'appelle et tu lui dis que tu n'y vas pas. Puis il appelle cinq autres amis et fatalement, tu finis toujours par y aller. (Majdak, 2007, notre traduction) ${ }^{16}$

La contagion est liée au phénomène de contrainte et de pression du groupe $^{17}$. Or, ce qui est intéressant dans le cas des Scorpions, et qui diffère du groupe des Tigres, où c'est davantage la discipline qui régulait les interactions entre les membres (Stewart, 2007), c'est que ce sont les liens $d u$ clan et la nature même de ces liens, quasi organique, qui expliquent les dynamiques d'influences mutuelles au sein de l'unité. La plupart des membres venaient du même village et se connaissaient depuis longtemps. Par exemple, à un moment donné, Dusko Kosanovic commente à la caméra des images qu'il a lui-même filmées. On y voit des membres de l'unité, habillés de leur tenue de combat noire, défiler devant un prêtre orthodoxe pour y recevoir les sacrements. Kosanovic explique:

Celui-ci, c'est le commandant en chef de la compagnie, le frère de Boco [Slobodan Medic], Guljo [Aleksandar Medic]. Ça, c'est l'adjoint, mon cousin Zoran Rakic. Ça, c'est mon pote, Slobodan Davidovic, qui a été accusé et reconnu coupable d'un crime, en Croatie. Celui-là était un des commandants du peloton de scouts. Voici Srecko, un jeune type qui a été tué à la fin, quand les Scorpions se sont désagrégés. Voici Pera Petrasevic, qui a été accusé de meurtre à Trnovo, et voici Branislav Medic, qui a été accusé de la même chose à Treskavica [dans la région de Sarajevo]. Voici un de nos amis d'école. Voici mon cousin Zlatko. Voici mon cousin Momic, qui a été condamné. Voici mon cousin Sico. Tout ça, en fait, constituait la force des Scorpions, tous les liens familiaux. Voici Goran Stoparic, nous avions tous des liens familiaux et étions des vieux amis de l'école. (Majdak, 2007, notre traduction)

16. Relevons que l'engagement de Goran Stoparic dans la violence tenait aussi d'une prise en considération du contexte politique et social plus vaste tel qu'il se manifestait dans cette région à cette époque:

À la télévision, vous pouviez voir des Croates hisser leur drapeau. Les Musulmans, ou si vous voulez, les Bosniaques hisser leur drapeau bosnien. Les Macédoniens le leur et les Serbes le leur. Et je me suis trouvé à l'endroit où mon drapeau était [...]. Il y avait une autre raison pour laquelle je suis parti combattre; vous savez très bien comment les médias ont présenté [les événements]. Vous vous enrôliez et vous partiez [simplement]. (Majdak, 2007, notre traduction)

17. Ce phénomène a fait l'objet d'une littérature abondante et on peut mentionner les travaux de Solomon Asch (1951) ou encore d'Henri Tajfel (1981). 
L'unité comptait au moins cinq cousins, deux frères, ainsi que des amis proches et de longue date qui se connaissaient depuis la petite école. Relevons aussi l'insistance de Kosanovic sur l'importance des liens familiaux qui faisaient la force de cette unité qui, avec l'amitié, confère sa pleine substance à la notion de clan. Ces individus étaient associés, agencés même, par une histoire, un passé, une famille, un lieu. Les échanges sociaux et leur régulation se jouaient et se déployaient sur une maille extrêmement serrée. C'est ce qui permet de saisir la citation ci-dessus de Goran Stoparic à sa pleine valeur. Le groupe, d'autant plus quand les liens sont aussi forts, et quasi organiques, est incontestablement une source majeure de constitution d'identité, de préférences, qui fournit un cadre référentiel, ou normatif, à ses membres (Goffman, 1974). Outre le fait que la responsabilité de l'individu s'y dissout - et facilite ainsi l'émergence de comportements radicaux -, ce cadre participe au maintien de l'«équilibre» de l'individu dans un environnement instable, qu'il soit politique, social, économique, lointain ou immédiat (Welzer, 2007). Il fournit une base commune de significations à partir de laquelle l'acteur donne sens aux événements qui l'entourent:

Ces références positives et négatives permettent à l'individu de ne pas rester seul avec ses idées et avec ce qu'on lui demande, mais de trouver sa place. Et c'est cette possibilité donnée à l'individu de se situer moralement et socialement dans le groupe qui assure l'efficacité meurtrière de ce dernier. (Welzer, 2007: 172)

Se soustraire du groupe par le refus de participer aux actions en cours entraîne des conséquences importantes pour l'individu, telles que l'ostracisme, et des effets identitaires que très peu sont prêts, ou capables, d'assumer. Ces résultats tendent à confirmer une hypothèse pourtant paradoxale à laquelle Christopher R. Browning (1992) était parvenu dans ses recherches sur les «hommes ordinaires» du $101^{\mathrm{e}}$ bataillon de la police de réserve. L'auteur avait affirmé que les tueries ne relevaient pas systématiquement d'une préférence individuelle des acteurs, mais aussi, et surtout, d'une décision liée à leur «survie sociale». Les individus, affirme Browning (1992), préfèrent tuer massivement et demeurer intégrés à ce qu'ils définissent comme leur groupe d'appartenance plutôt que de faire le choix de la dissidence et épargner des vies. Ils ne sont pas moins responsables de leurs actes. Les individus sont engagés dans des groupes sociaux et on ne peut faire fi des dynamiques qui s'y jouent et tracent l'espace des possibles/impossibles, où 
l'action est envisageable pour eux. Ces dynamiques informent tout autant le cadre normatif des membres. À leur tour, ces groupes, comme le rappelle Martin Shaw (2007), évoluent dans des contingences politiques et sociales, provoquant le bouleversement des cadres qui informent les identités, significations et références, et qui sont à la base de l'action des membres.

Cette perspective entraîne un bouleversement important d'un point de vue conceptuel et en matière de compréhension et d'explication de la participation d'individus dans des actions de violence de masse. Si, tel que le prétend la sociologie réflexive qui sous-tend les conjectures de Welzer (2007) ou Shaw (2007), l'action individuelle est le produit d'une interprétation de l'environnement par les acteurs, alors l'intention, ou la décision de commettre l'action violente ne doit plus tant s'envisager comme une variable indépendante, mais plutôt comme une question empirique per se et une variable dépendante. Dans le cadre des membres de l'unité des Scorpions, et cet argument est tout autant valable pour les hommes ordinaires analysés par Browning (1992), la déviance et les actes criminels des individus s'interprètent comme le produit d'un processus ou d'une série de mécanismes qui bouleversent le cadre normatif, ou de référence, des acteurs. Ce cadre interprétatif s'actualise au fur et à mesure de la progression des événements politiques et sociaux qui entourent les acteurs - et les bandes armées - et produit de la nouveauté psychique qui, progressivement, et selon le principe du point de basculement, non seulement permet l'action radicale, mais aussi métamorphose la représentation des actes criminels en action sociale normativement adéquate. C'est en cela que la nécessité de tenir compte du groupe, de la bande armée et des dynamiques qui caractérisent son fonctionnement interne se justifie pour saisir, du moins en partie, la genèse de la criminalité de masse.

\section{Vers une compréhension alternative de la participation des exécuteurs?}

Il ne fait aucun doute qu'un lien de collusion a caractérisé les relations entre les Scorpions et le pouvoir central serbe. Or, il est erroné de croire que le mode de participation de cette bande armée se caractérise par une dynamique de «sélection préférentielle» (Valentino, 2004), ou par un «mécanisme relativement banal» (Mueller, 2004). Ces conjectures renvoient au principe selon lequel un État recrute, en vertu d'un modèle 
décisionnel simple, autant de psychopathes, sadiques, maraudeurs et fanatiques, auxquels on donne les coudées franches, et on mise sur leur potentiel de destruction pour faire fuir les populations civiles. Il ne faudrait toutefois pas en conclure que ces unités n'ont pas également hébergé ce type d'individus, comme l'indique Goran Stoparic à quelques reprises dans son témoignage.

Ce que nous avons plutôt démontré, c'est le rôle crucial joué par un niveau d'organisation intermédiaire, entre pouvoir central et individu, à savoir le groupe et la bande armée, et la nécessité de considérer la dimension collective de ce type de criminalité. Épistémologiquement, la prise en compte de cette unité d'analyse laisse entendre que l'action individuelle est hautement tributaire des pratiques du groupe lui-même et des complicités, ou influences, qui s'y trament, mais aussi du contexte social et politique dans lequel l'unité évolue. Ceci ne tient pas tant strictement aux mécanismes de pression à la conformité, comme une longue tradition de recherche l'a abondamment montré, mais à un phénomène identitaire et à un jeu de positionnement des individus tout au long de leur carrière, ou trajectoire d'exécuteur au sein du groupe qui leur sert de référent. Ces unités participent à la métamorphose de l'image que ces acteurs ont d'eux-mêmes et provoquent une «redistribution des repères de la moralité» (Banégas et Warnier, 2001: 8). Il faut donc porter une attention toute particulière à l'évolution de ces modes de subjectivations de soi et du cadre normatif qui sous-tendent les actions et pratiques sociales des protagonistes. Dans ces conditions, l'intention, envisagée comme variable dépendante, émerge et se développe au gré des circonstances et des réseaux d'acteurs dans lesquels les protagonistes sont insérés. Ces modes de subjectivation de soi produisent une nouveauté qualitative dans la manière qu'a l'individu d'interpréter son environnement. Comme nous l'avons documenté dans d'autres contextes (Tanner, 2011), cette nouveauté est le produit d'une séquence, plus ou moins longue, de décisions à la pièce qui doivent être documentées et analysées.

Les dynamiques qui animent les bandes armées permettent d'envisager la collusion qu'elles entretiennent avec le pouvoir central sous un regard nouveau. S'il est incontestable que ce dernier ouvre la voie, par la promotion d'un discours de haine et d'un ensemble de décisions politiques affichant ouvertement une hostilité à l'encontre du horsgroupe, ou qu'il cautionne ou entérine la violence et qu'il fournit les ressources matérielles à ces unités, les pratiques radicales des bandes 
armées ne peuvent s'expliquer uniquement par une approche verticale, en vertu de laquelle l'État assurerait l'ensemble du commandement, du contrôle, du déploiement de la violence et de la destruction des populations. Dans l'esprit de l'approche de Ian Kershaw (1998), il est nécessaire d'intégrer les jeux de pouvoir, de concurrence et d'autonomisation, tels qu'ils s'articulent entre les acteurs constitutifs du dispositif d'élimination de la population et les animent. Ces logiques sociales relèvent des rapports de force qui incitent, suscitent et combinent les acteurs entre eux et, ultimement, les font basculer dans la violence. Celle-ci n'est alors pas tant le fait d'une appropriation par un acteur exclusif, l'État, mais plutôt de dispositions (la défense de la quiddité serbe), de manœuvres (l'accumulation de ressources), de tactiques (la définition d'objectifs en termes vagues, ou généraux et laissant place à l'interprétation), de techniques (la violence) et de fonctionnements (la discipline interne, la régulation par les liens du clan). La violence s'exerce plutôt qu'elle ne se possède, elle n'est pas le privilège acquis, ou conservé, du pouvoir central, mais l'effet d'ensemble des protagonistes qui l'exercent et des positions qu'ils détiennent au sein du dispositif qui la sous-tend ${ }^{18}$.

Enfin, cette analyse permet d'envisager une définition plus substantielle de la bande armée. En premier lieu, elle se compose de volontaires et d'entrepreneurs. Ces sont des acteurs capables de mettre sur pied des solutions organisationnelles et d'élaborer des actions stratégiques, permettant la conversion d'un type de ressources (l'usage de la force, les profits générés par la participation à une activité criminelle) en d'autres types de bénéfices, ou profits prisés, et ce, sur une base permanente $^{19}$. Deuxièmement, la bande armée est un concept heuristique qui renvoie à des groupes d'exécuteurs dont les dynamiques de participation aux violences sont complexes. Leur genèse tient d'un jeu d'interactions entre acteurs périphériques et régime central. Elles résultent d'une cristallisation d'un rapport de force qui se joue dans un champ hybride mêlant criminalité de faible, moyenne et longue portée. En conséquence, les bandes armées sont une figure centrale de cette zone de métissage de la criminalité de guerre qui tend à politiser une criminalité civile et à privatiser une criminalité politique. Les pratiques sous-

18. Cette réflexion est inspirée des propos de Michel Foucault sur le pouvoir, dans Foucault (1976).

19. Cette définition est inspirée des travaux de Vadim Volkov sur les entrepreneurs de la violence dans le contexte de la mafia russe (Volkov, 2002). 
tendant le projet politique de nettoyage ethnique sont banalisées comme autant de faits de groupes criminels locaux et sur lesquels le pouvoir central n'exercerait aucun contrôle. Enfin, les bandes armées s'envisagent dans leur dimension communautaire. Si elles tirent profit d'un «espace d'autorisation» créé par le pouvoir central, c'est également dans la sphère et les réseaux sociaux locaux, qui facilitent leur participation, que leur sort se joue. Ainsi, la bande armée est un concept dynamique qui nécessite d'être saisi non pas tant et strictement en vertu de ses actions finalisées (perspective téléologique), c'est-à-dire par les conséquences de sa participation, mais bien aussi dans son processus de genèse, d'émergence, de mobilisation, de déploiement. C'est précisément cette démarche qui nous a permis d'envisager comment une unité de sécurité privée s'est, peu à peu, transformée en armée de destruction massive.

\section{Références}

Anastasijevic, D. (2005). The Trail of the Scorpions. News and Analysis series, 45-46 (mai-août). Bosnian Institute. Disponible sur: www.bosnia.org.uk/ news/news_body.cfm? newsid=2075

Arendt, H. (1964). Eichmann à Jérusalem: rapport sur la banalité du mal. Paris: Gallimard (édition de 1966).

Arendt, H. (2003). Responsabilité et jugement. Paris: Payot (traduction, édition de 2005).

Asch, S. (1951). Effects of Group Pressure Upon the Modification and Distortion of Judgements. In H. Guetzkow (Ed.), Groups, Leadership and Men (177-190). Pittsburgh: Carnegie Press.

Banégas, R., \& Warnier, J. P. (2001). Nouvelles figures de la réussite et du pouvoir. Dossier intitulé Figures de la réussite et imaginaires politiques. Politique Africaine, 82 (juin), 5-23, 8.

Bauman, Z. (1989). Modernity and the Holocaust. Ithaca, NY: Cornell University Press.

Becker, H. S. (1985). Outsiders. Études de sociologie de la déviance. Paris: Métailié.

Brodeur, J.-P. (2007). De grandes espérances. Criminologie, 40 (2), 161-166.

Browning, C. R. (1992). Ordinary Men: Reserve Police Battalion 101 and the Final Solution in Poland. New York: Harper Collins.

Browning, C. R. (2007). Les origines de la Solution finale. L'évolution de la politique antijuive des nazis, septembre 1939 - mars 1942. Paris: Les Belles Lettres.

Deleuze, G. (1986). Foucault. Paris: Les Éditions de Minuit. 
Drakulic, S. (2004). They Would Not Hurt a Fly: War Criminals on Trial in The Hague. Londres: Penguin.

Foucault, M. (1976). Histoire de la sexualité 1. La volonté de savoir. Paris: Gallimard.

Fowler, M. C. (2005). Amateurs Soldiers, Global Wars. Insurgency and Modern Conflict. Westport, Connecticut; Londres: Praeger Security International.

Goffman, E. (1974). Frame Analysis: An Essay on the Organization of Experience. Boston: Northeastern University Press.

Judah, T. (1997). The Serbs: Myth, History and the Destruction of Yugoslavia. New Haven: Yale University Press.

Kershaw, I. (1998). Hitler: 1889-1936: Hubris. Paris: Flammarion.

Leman-Langlois, S. (2006). Le «mégacrime», légitimité, légalité et obéissance. Criminologie, 39 (2), 23-37.

Majdak, N. (2007). The Scorpions: A Home Movie. Belgrade, Serbie: Humanitarian Law Center.

Mommsen, H. (1989). The Realization of the Unthinkable: The Final Solution of the Jewish Question in the Third Reich. In M. Marrus (Ed.), The Nazi Holocaust. Historical Articles on the Destruction of European Jews (3, 217-264). Londres: Meckler.

Mueller, J. E. (2004). The Remnants of War. Ithaca, NY: Cornell University Press.

Nations Unies (1992). Résolution 743 du Conseil de sécurité sur la création d'une force de protection des Nations Unies (FORPRONU) en Yougoslavie, 21 février.

Petersen, R. D. (2001). Resistance and Rebellion: Lessons from Eastern Europe. Cambridge, UK; New York: Cambridge University Press.

Schulz, R. H., \& Dew, A. J. (2006). Insurgents, Terrorists and Militias: The Warriors of Contemporary Combat. New York: Columbia University Press.

Shaw, M. (2007). What Is Genocide? Cambridge; Malden, Mass. : Polity Press.

Stewart, C. S. (2007). Hunting the Tiger. The Fast Life and Violent Death of the Balkans' Most Dangerous Man. New York: St Martin's Press.

Tajfel, H. (1981). Human Groups and Social Categories. New York: Cambridge University Press.

Tanner, S. (2011). Towards a Pattern in Mass Violence Participation? An Analysis of Rwandan Perpatrators' Account from the 1994 Genocide. Global Crime (à paraître).

Tribunal pénal international pour l'ex-Yougoslavie (2003). Le Procureur $d u$ Tribunal contre Slobodan Milosević, Affaire IT-02-54, 21 avril 2004, audience du 14 octobre.

Valentino, B. A. (2004). Final Solutions, Mass Killing and Genocide in the Twentieth Century. Ithaca, NY: Cornell University Press.

Volkov, V. (2002). Violent Entrepreneurs: The Use of Force in the Making of Russian Capitalism. Ithaca: Cornell University Press.

War Crimes Chamber (2006). Slobodan Metic et al., Belgrade District Court, Republic of Serbia, Case K.br. 6-05, retranscription d'audiences couvrant la période du 20 décembre 2005 au 5 juillet 2006. 
War Crimes Prosecutor's Office (2005). The Prosecutor of the Tribunal vs Slobodan Metic et al., Republic of Serbia, Case KTRZ, br. 3/05. Belgrade, 7 octobre.

Weber, M. (1921). Économie et société, Vol. 1 : les catégories de la sociologie, Paris: Plon (édition de 1985).

Weinstein, J. (2007). Inside Rebellion: The Politics of Insurgent Violence. Cambridge, UK; New York: Cambridge University Press.

Welzer, H. (2007). Les exécuteurs: des hommes normaux aux meurtriers de masse. Paris: Gallimard.

ABSTRACT - This article focuses on how a small armed band - the Scorpions - participated in the events that occurred in former Yugoslavia. Considering the role and responsibility of such small units in one of the most radical destruction of humankind is not only necessary to understand and, ultimately prevent such tragedies. It is also justified on an epistemological basis. Rather than considering such participation through the individual and motivational aspect, which is a common perspective in main stream criminology, focusing on western societies' security preoccupations, I argue that grasping the dynamics underlying the destruction of entire populations necessitates to consider the collective and political nature of such violence, and therefore to focus on the group. Hopefully, such approach will help producing a more accurate knowledge, and thus a new look on mass crimes.

KEYWORDS • Mass violence, former Yugoslavia, Scorpions, Armed Bands, Radicalization, Private Security.

RESUMEN - El presente artículo analiza la movilización y participación de una banda armada - los Escorpiones - en los eventos de ex Yugoslavia en el decenio de 1990. Si el estudio de esta forma radical de destrucción del hombre es necesario para su comprensión y, en última instancia, su prevención, también se justifica desde el punto de vista epistemológico: en lugar de centrar la reflexión en el individuo y sus motivaciones, enfoque común en la criminología dominante centrada en las preocupaciones sobre seguridad de las sociedades occidentales, postulamos que es a partir del grupo y de la naturaleza eminentemente colectiva y política de esta forma de criminalidad extrema que debe realizarse la reflexión sobre las dinámicas de eliminación de poblaciones enteras. Este enfoque permite generar conocimientos más específicos y una perspectiva nueva sobre la criminalidad de masa.

PALABRAS CLAVE - Violencia de masa, ex Yugoslavia, Escorpiones, bandas armadas, radicalización, seguridad privada. 